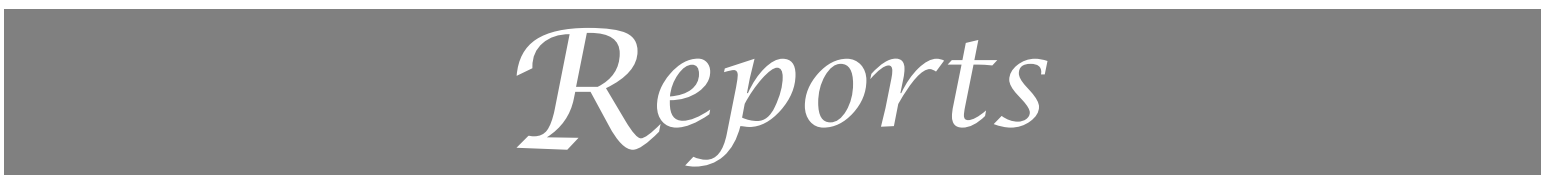

Ecology, 96(7), 2015, pp. 1754-1760

(c) 2015 by the Ecological Society of America

\title{
Phenological synchronization drives demographic rates of populations
}

\author{
Nick L. Rasmussen ${ }^{1}$ and Volker H. W. Rudolf \\ Department of Ecology and Evolutionary Biology, Rice University, Houston, Texas 77005 USA
}

\begin{abstract}
Phenology is increasingly recognized as an important factor structuring communities because it determines when and at what life stage organisms interact. Previous work indicates that changes in first or mean timing of a phenological event can affect populations and communities, but little is known about the consequences of changes in the distribution (e.g., synchrony) of a phenological event. We conducted an experiment using an anuran study system to determine how synchrony of reproduction and egg hatching affects offspring performance, whether the effects are density dependent, and how hatching synchrony influences the synchrony of a subsequent phenological event (metamorphosis). Changes in hatching synchrony altered survival, development rates, and body size at metamorphosis, which can affect post-metamorphosis performance. The degree of synchrony at hatching also affected the degree of synchrony at metamorphosis, indicating that timing of one stage can carry over to affect that of later ones. Importantly, these effects were all density dependent, likely because decreasing hatching synchrony switched intraspecific interactions from scramble to contest competition. This study demonstrates that phenological synchrony has important consequences for ecological interactions and population dynamics, emphasizing the need to develop a comprehensive understanding of how shifts in phenological distributions affect communities.
\end{abstract}

Key words: Bufo nebulifer; climate change; coastal-plain toad; competition; density dependence; metamorphosis; priority effects; seasonal dynamics; synchrony; tadpole.

\section{INTRODUCTION}

Phenology, the seasonal timing of life-history events, determines when during the year organisms interact with both conspecifics and heterospecifics. Phenologies can, however, vary substantially among years, and climate change is altering phenologies of species worldwide (Parmesan 2007, Miller-Rushing et al. 2010). Previous work has demonstrated that shifts in the first or mean timing of a phenological event can alter species interactions and population dynamics (reviewed by Miller-Rushing et al. 2010). While these studies emphasize the importance of phenologies in structuring natural communities, this approach neglects the fact that a phenological event exhibits a temporal distribution, the shape of which is determined by the timing of all the individuals within a population (Miller-Rushing et al.

Manuscript received 7 October 2014; revised 28 January 2015; accepted 19 March 2015. Corresponding Editor: C. E. Cáceres.

${ }^{1}$ E-mail: solifugae@gmail.com
2010, CaraDonna et al. 2014). For example, the degree of synchrony among individuals within a population (i.e., amount of variation in timing around the mean) can vary due to interannual variation in seasonal weather patterns and differential responses by individuals to it (Blair 1960, Ogutu et al. 2010). While the degree of synchrony likely has important implications for intraspecific interactions, we still have a poor understanding of when and how it affects populations (Miller-Rushing et al. 2010).

There are many ways in which synchrony could affect intraspecific interactions. The degree of synchrony of offspring production, for example, determines the number of offspring that co-occur in time (increases with higher synchrony), as well as the amount of body size variation of offspring (decreases with higher synchrony). Density and relative body size are important factors determining the strength and type of intraspecific priority effects that occur among offspring (Hopper et al. 1996, Sunahara and Mogi 2002, Eitam et al. 2005, Geange and Stier 2009). When synchrony of 
offspring production is high, offspring are similar in size when competition is initiated, which likely results in symmetric competition (i.e., scramble competition). In contrast, when offspring production occurs asynchronously, this creates variation in age/size among offspring, which could result in asymmetric competition (i.e., contest competition), favoring older/larger individuals. Effects of synchrony of offspring production are likely density dependent, with variation in synchrony having stronger effects on demographic rates at higher densities. At low densities, per capita resource availability, and thus survival, is likely high regardless of timing. However, at high densities, the dynamics of low- vs. high-synchrony cohorts could differ in interesting ways. If high synchrony causes symmetric competition, then survival could be high, at least initially, because it is difficult for any individual to gain an advantage. The resulting low per capita resource availability could cause prolonged development and small body size at maturity for cohort members. If low synchrony causes asymmetric competition, then high density could cause intense priority effects (Hopper et al. 1996). The early-arriving offspring could dominate resources and complete development quickly and at a large size, which would likely result in high mortality of later-arriving offspring. Therefore, synchrony and density likely interact to affect survival and other demographic rates, such as development rates and body size, through effects on competition.

In addition to altering demographic rates, it is also important to consider how phenological synchrony at one stage carries over to influence that of subsequent life stages. For example, while the degree of synchrony of one stage could be perpetuated to subsequent phenological events (Augspurger 1981), this may not always be the case. Synchrony of subsequent events could increase if individuals born at one or both tails of the temporal distribution suffer high mortality (Hopper et al. 1996). Conversely, synchrony of subsequent events could decrease due to intraspecific variation in development rates. Ultimately, the nature of this inter-stage change in phenological synchrony likely depends upon the ecological context, such as intensity and type of competition, but to our knowledge, this has not been examined.

Here, we took an experimental approach using an anuran study system to understand how synchrony of offspring birth affects demographic rates. Specifically, we answered the following three questions: (1) how does variation in synchrony of hatching affect demographic rates, (2) are the effects density dependent, and (3) how does the synchrony of hatching affect the synchrony of subsequent phenological events (i.e., metamorphosis)? In general, we found that changes in synchrony can alter demographic rates and synchrony of later phenological events, but these effects also depend upon the ecological context (i.e., density).

\section{Methods}

\section{Study system}

We studied the coastal-plain toad (Bufo nebulifer) to determine the consequences of variation in hatching synchrony for the performance of cohorts of offspring. The species is common throughout much of the Gulf Coastal Plain, including eastern Texas, USA. It breeds primarily in ephemeral pools created by summer rains, and the amount and duration of reproductive activity varies among years with changes in rainfall patterns (Blair 1960; N. L. Rasmussen, personal observation). Consequently, the synchrony of reproduction, and subsequent tadpole hatching, can range from high (occurring on a single day) to low (extending over a week). Hatching patterns are important for this species because the habitats are small and ephemeral, nutrients are limited, and tadpole densities can be very high. These factors create a highly competitive environment. Therefore, a clutch hatching even one day earlier than others in a pool can have a significant advantage. Specifically, early-hatching tadpoles can gain a body size advantage and monopolize food resources prior to colonization by later-hatching ones (i.e., generate priority effects; Wilbur and Alford 1985, Rudolf and Singh 2013). This promotes growth of the early tadpoles while reducing that of late ones, and this can lead to a positive feedback between size and growth rates. This effect of hatching timing on the symmetry of competition among cohorts could have important implications not only for the number of tadpoles that survive to metamorphosis but also mean body size at metamorphosis, mean development time, and the degree of synchrony of metamorphosis, all of which can affect post-metamorphosis performance (Wilbur 1997). In the long term, phenological synchrony could affect population dynamics by altering the number and quality of individuals recruiting into the adult population annually (Domínguez and Dirzo 1995).

\section{Experimental design and setup}

To create the synchrony treatments, we manipulated the amount of temporal variation in tadpole hatching around a mean hatching date (28 June 2011). In the high-synchrony treatment, all tadpoles were hatched on the mean date (no temporal variation in hatching). For both the medium- and low-synchrony treatments, hatching was evenly divided among three dates. This included the mean date as well as one date before and one date after the mean date (medium, mean $\pm 2 \mathrm{~d}$; low, mean $\pm 3 \mathrm{~d}$ ). These manipulations capture most of the variation in hatching synchrony that we observe in nature (i.e., hatching duration of one day to one week). To determine whether variation in hatching synchrony could alter density-dependent competition, we crossed these three levels of synchrony with five levels of initial density $(60,75,90,120,180$ tadpoles). Each of these 15 treatment combinations was replicated three times in a 
randomized complete-block design (45 experimental units total).

On the night of 22 June 2011, we collected four amplectic pairs of $B$. nebulifer from ephemeral pools in Houston, Texas. The following morning, each of the resulting egg clutches was divided into many short sections ( $\sim 300$ eggs), which were evenly distributed among five groups. Consequently, the five groups contained about the same proportion of eggs from each clutch (i.e., genetic composition was similar). Each group was incubated at a different temperature along a thermal gradient $\left(20-31^{\circ} \mathrm{C}\right)$. These temperature treatments allowed us to manipulate development rates, so hatchlings (Gosner stage 25) would be available on the five dates required to create the three synchrony treatments. It is possible that these temperature manipulations created differences among cohorts, but we expect these differences were minimal. This is because $B$. nebulifer eggs naturally develop in ephemeral pools, which vary tremendously in temperature. Also, our temperature gradient was well within that which Ballinger and McKinney (1966) demonstrated to promote high survival and normal development of $B$. nebulifer eggs. As tadpoles in each group hatched, they were haphazardly added to the appropriate pools.

The experiment was conducted in an open field in Houston. We used an array of 45 plastic pools $(100 \mathrm{~cm}$ diameter, $19 \mathrm{~cm}$ depth) arranged into three rectangular spatial blocks. Within these containers, we simulated natural pools. We filled them with dechlorinated water on 20 June and immediately fitted them with lids made of $60 \%$ shade cloth to prevent unwanted colonization by predatory insects and oviposition by other amphibians. We added to each pool $1 \mathrm{~L}$ of concentrated phytoplankton and zooplankton from local pools (22 June), $700 \mathrm{~mL}$ wet mass of live vegetation (24 June), and $37 \mathrm{~g}$ air-dried mass of leaf litter (24 June). Hatchlings were added from 25 June to 1 July. Pools were monitored daily to collect metamorphs. Metamorphs were weighed after their tails were completely absorbed. Then, they were released near the site where adults had been captured. The experiment was ended on 16 August, seven weeks after the mean hatching date (maximum persistence of local pools; N. L. Rasmussen, personal observation).

We used four responses to determine the consequences of our treatments on performance of offspring cohorts: mean proportion of survivors (number of metamorphs/initial number of hatchlings), mean metamorph biomass (total wet mass of metamorphs/number of metamorphs), mean development time (total days of larval development summed across metamorphs/number of metamorphs), and inter-stage change in phenological synchrony $\left(\Delta \mathrm{CV}=\mathrm{CV}_{\mathrm{m}}-\mathrm{CV}_{\mathrm{h}}\right.$; difference between coefficient of variation for time to metamorphosis $\left[\mathrm{CV}_{\mathrm{m}}\right]$ and coefficient of variation for hatching $\left.\left[\mathrm{CV}_{\mathrm{h}}\right]\right)$. For proportion of survivors, only individuals that achieved metamorphosis during the experiment (seven-week period) were counted as survivors. Many pools in the experiment still contained tadpoles at the end of the experiment, and the number of these varied by treatment (see Results: Survival). However, ephemeral pools rarely persist longer than seven weeks, so these tadpoles would have perished in nature. Continuing the experiment until all tadpoles either died or metamorphosed likely would have altered all the responses measured, but given that the time required for this would represent a highly unrealistic situation, it would be unclear how to interpret those results. For mean development time, development time for each individual was calculated as the difference between its date of metamorphosis and the mean hatching date, because it was not possible to determine which metamorphs were derived from which hatching dates. For the inter-stage change in synchrony, positive, negative, and zero values would indicate an increase, decrease, and no change in phenological variation, respectively. As the synchrony treatments were set up, $\mathrm{CV}_{\mathrm{h}}$ in the low-, medium-, and high-synchrony treatments was $0.62,0.41$, and 0.0 , respectively. Therefore, high-synchrony treatments could only exhibit no change or an increase in variation. Medium- and low-synchrony treatments, however, could increase, decrease, or show no change. For amphibians, size at metamorphosis is often positively correlated with future survival, size at maturity, and/or date of first reproduction (Semlitsch et al. 1988, Berven 1990, Goater 1994). Development time is often negatively correlated with future survival (Berven 1990) and/or size at maturity (Semlitsch et al. 1988).

\section{Statistical analysis}

All analyses were performed in the $\mathrm{R}$ statistical computing environment ( $\mathrm{R}$ Development Core Team 2013). Using the lme4 package (Bates et al. 2013), we ran generalized linear mixed models to analyze the effects of variation in hatching synchrony (categorical predictor with three levels) and initial density (treated as a continuous predictor) on proportion of survivors, mean metamorph biomass, mean development time, and interstage change in synchrony. Plots of each of these latter three responses vs. density suggested a nonlinear relationship, so a quadratic term for density was added to full models for all three analyses. Inclusion of the quadratic term is supported by previous studies that have determined that metamorph mass and development time nonlinearly decreases and increases, respectively, with increasing density (Wilbur 1997). Unless otherwise noted, spatial block was the only random effect. To analyze proportion of survivors, we used a binomial error structure and included an observation-level random effect to deal with overdispersion (Zuur et al. 2013). To analyze the other three responses, we used a Gaussian error structure. For all four analyses, we evaluated significance of fixed effects and their interactions by generating analysis of variance/deviance tables 

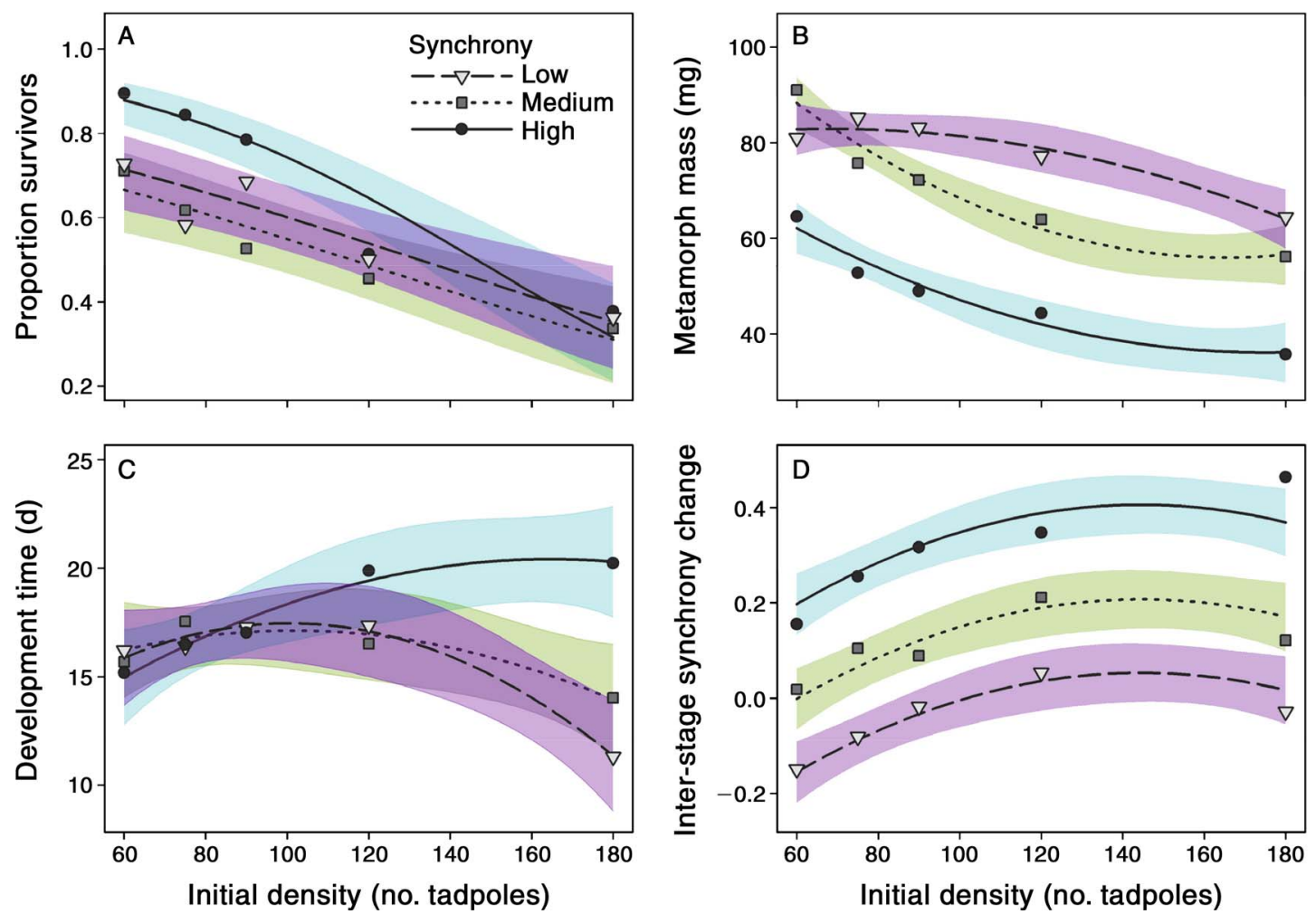

FIG. 1. Responses of cohorts of Bufo nebulifer to experimental manipulations of hatching synchrony (high, medium, and low) and initial density $(60,75,90,120$, and 180 tadpoles). (A) Mean proportion of survivors, (B) mean metamorph mass, (C) mean development time, and (D) change in phenological synchrony (change in coefficient of variation [CV]) between egg hatching $\left(\mathrm{CV}_{\mathrm{h}}\right)$ and metamorphosis $\left(\mathrm{CV}_{\mathrm{m}}\right)$. Fitted lines and $95 \%$ confidence intervals (represented by color bands) were generated from generalized linear mixed models. Points are the means for the raw data (three replicates per treatment combination).

with the car package (Fox and Weisberg 2011) and removed nonsignificant terms from final models $(P>$ 0.05). To test for significance of the random effect (spatial block), we used Akaike's information criterion (AIC) to compare models containing no random term, except for the ordinary residuals, to models with the random intercept (Zuur et al. 2013).

\section{REsults \\ Survival}

Proportion of tadpoles surviving to metamorphosis was driven by the interaction of hatching synchrony and density (Fig. 1A; synchrony, $\chi^{2}=16.36, P=0.0003$; density, $\chi^{2}=75.53, P<0.0001$; synchrony $\times$ density, $\chi^{2}$ $=7.01, P=0.0300$ ). Proportion of survivors declined as density increased (from 0.78 at the lowest density to 0.36 at the highest density, ignoring synchrony levels), but the relationship for high synchrony differed from that of medium and low synchrony. Specifically, at low densities, proportion of survivors in the high-synchrony treatment was $20 \%$ and $19 \%$ higher than that in the medium- and low-synchrony treatments, respectively, but survival in all synchrony treatments converged at high densities.
Many pools (63\%) contained tadpoles at the end of the experiment, and the mean number of tadpoles per pool varied by treatment (low synchrony, density ${ }_{60}=0.0$, density $_{75}=0.3$, density $y_{90}=6.0$, density $y_{120}=14.7$, density ${ }_{180}$ $=9.3$; medium synchrony, density ${ }_{60}=0.0$, density ${ }_{75}=5.3$, density $_{90}=17.0$, density $_{120}=31.0$, density $_{180}=17.0$; high synchrony, density ${ }_{60}=0.0$, density $y_{75}=0.7$, density ${ }_{90}=4.0$, density $_{120}=4.3$, density $\left.y_{180}=40.7\right)$. However, they would have died in natural pools, which rarely persist for more than seven weeks (duration of the experiment).

\section{Metamorph biomass}

Mean mass was driven by the interaction of synchrony and density (Fig. 1B; synchrony, $F_{2,36}=121.25, P<$ 0.0001 ; density + density $^{2}, F_{2,36}=63.54, P<0.0001$; synchrony $\times\left[\right.$ density + density $\left.^{2}\right], F_{4,36}=3.88, P=$ 0.0101 ). Mean mass declined nonlinearly with density (from $78.8 \mathrm{mg}$ at the lowest density to $52.0 \mathrm{mg}$ at the highest density, ignoring synchrony levels), but the relationship differed among synchrony levels. Specifically, mean mass was lowest for the high-synchrony treatment across all densities $(69 \%$ and $63 \%$ that of medium- and low-synchrony treatments, respectively, ignoring density levels). Medium- and low-synchrony treatments exhibited mean masses similar to one another 
at the lowest and highest densities but diverged at intermediate densities, owing to differences in the shape of the density-dependent relationship (medium synchrony was concave, low synchrony was convex).

\section{Development time}

Development time was determined by the interaction of synchrony and density (Fig. $1 C$; synchrony, $F_{2,36}=3.59$, $P=0.0378$; density + density $^{2}, F_{2,36}=4.48, P=0.0183$; synchrony $\times\left[\right.$ density + density $\left.\left.^{2}\right], F_{4,36}=5.57, P=0.0013\right)$. At the lowest density, development time was similar across synchrony treatments $(15.7 \mathrm{~d})$, but synchrony treatments differed in their density-dependent relationships and diverged at high densities. For high synchrony, development time increased nonlinearly with density, while it decreased nonlinearly for medium- and lowsynchrony treatments. Consequently, at the highest density, development time with high synchrony was $31 \%$ and $44 \%$ longer than that with medium and low synchrony, respectively.

\section{Inter-stage synchrony change}

The change in synchrony $(\triangle \mathrm{CV})$ between hatching $\left(\mathrm{CV}_{\mathrm{h}}\right)$ and metamorphosis $\left(\mathrm{CV}_{\mathrm{m}}\right)$ depended upon synchrony and density but not their interaction (Fig. $1 \mathrm{D}$; synchrony, $F_{2,40}=58.06, P<0.0001$; density + density $\left.^{2}, F_{2,40}=13.65, P<0.0001\right) . \Delta \mathrm{CV}$ increased nonlinearly with density across all synchrony levels. High synchrony showed a positive $\Delta \mathrm{CV}$ across all densities (i.e., increase in variation between hatching and metamorphosis). Medium synchrony showed a $\Delta \mathrm{CV}$ of about zero at the lowest density and a positive $\Delta \mathrm{CV}$ at higher densities. For low synchrony, $\Delta \mathrm{CV}$ was negative at low densities (i.e., decrease in variation between hatching and metamorphosis) and reached zero at high densities. This indicates that synchrony of one phenological event can influence that of subsequent events, even across a range of densities.

\section{Discussion}

Though variation in phenological synchrony is widespread, we know little about the consequences for populations. Using an empirical system, we found that synchrony of offspring birth affected density-dependent competition in ways that altered several important attributes of the resulting cohorts of juveniles. These results indicate that hatching synchrony can determine the number, quality, and timing of offspring recruiting into the adult population, but understanding the specific outcome requires knowledge of the ecological context (e.g., density).

\section{Effects of synchrony on competition dynamics}

While the interaction of synchrony and density on attributes of juvenile cohorts may seem complicated, this is consistent with the hypothesis that hatching synchrony can alter the type of density-dependent competition (i.e., shift from scramble competition at high synchrony to contest competition at low synchrony). With high synchrony, tadpoles were similar in age/size (and thus competitive abilities) when interactions were initiated, which should lead to scramble competition. At low densities, a high proportion of tadpoles survived to metamorphosis but had low average mass (Fig. 1, solid line), which corroborates the idea that competitive similarity made it difficult for any tadpoles to gain an advantage and thus forced them to partition food resources equally. At high densities (and competition intensities), tadpole numbers exceeded resource carrying capacity, so a high proportion gradually starved to death. This likely explains why metamorphs exhibited prolonged development and low mass (Fig. 1, solid line).

As synchrony shifted to lower levels, competition likely changed from scramble to contest competition due to priority effects. In the low-synchrony treatment, the first cohort of tadpoles hatched six days before the third one (six days is $\sim 50 \%$ of development time under ideal conditions). Consequently, tadpoles in this first cohort likely experienced higher per capita resource levels (compared to high synchrony), which they monopolized and used to achieve a size-mediated competitive advantage over later-arriving cohorts. Due to this asymmetric competition, mortality in the two laterhatching cohorts should have been high and occurred early in development. At low densities, this low survival (relative to high synchrony) allowed those that achieved metamorphosis to do so quickly and at a large mass (Fig. 1, dashed line). At high densities, negative effects of the first cohort on later cohorts would have been magnified, likely further increasing mortality for laterhatching cohorts and increasing the proportion of metamorphs from the first cohort. This could partially explain the reduction in mean development time with increasing density for medium- and low-synchrony treatments, despite the absolute number of metamorphs increasing (as opposed to proportion of survivors, which decreased) and mean mass decreasing (Fig. 1, dashed line). This could occur because development time was calculated as the time difference between mean hatching date and metamorphosis date, which underestimates development time of tadpoles that hatched before the mean hatching date (first cohort). Our calculation of mean development time, however, cannot completely explain the differences in development time among synchrony levels at high densities. The difference in arrival time between tadpoles in the high-synchrony treatment and the first cohort in the low-synchrony treatment was only three days, while the difference in mean development time between these treatments was nine days. These findings are consistent with prior work in other systems, which indicates that early-arriving cohorts can have strong negative effects on later-arriving ones (Hopper et al. 1996, Sunahara and Mogi 2002, Eitam et al. 2005). Our results also indicate that synchrony can alter the type of density-dependent competition and its effects on populations. 


\section{Maintenance of phenological synchrony across life-history events}

Synchrony of one phenological event can affect not only the success of that particular event but also synchrony of subsequent events. For example, synchrony of flowering determines not only success of pollination but also synchrony of subsequent seed maturation, which affects losses to seed predators (Augspurger 1981). Therefore, it is important to understand the cascade of effects due to phenological synchrony. In our experiment, we found that the different synchrony levels converged somewhat between hatching and metamorphosis (i.e., high-synchrony treatments, $\mathrm{CV}_{\mathrm{h}}<\mathrm{CV}_{\mathrm{m}}$; low-synchrony treatments, $\mathrm{CV}_{\mathrm{h}}>\mathrm{CV}_{\mathrm{m}}$; Fig. 1D). Nonetheless, these synchrony levels remained distinctly different from one another in terms of $\mathrm{CV}$ at all densities, indicating a persistent signature of variation in synchrony from one phenological event to the next one. We also found, for all levels of hatching synchrony, that synchrony of metamorphosis decreased with increasing density. This is consistent with densitydependent competition, which would magnify the differences among individuals in traits related to development rate, such as activity levels or food conversion efficiency (Wilbur 1997).

Variation in metamorphosis synchrony due to variation in hatching synchrony has important ecological implications. Many toad species exhibit highly synchronized metamorphosis as an anti-predator defense (DeVito 2003). If the majority of individuals develop through this vulnerable life stage at once, predators become "swamped" by available prey, resulting in a high proportion of toads surviving to a less vulnerable life stage. This means that hatching synchrony could alter the effectiveness of this anti-predator strategy and ultimately recruitment of juveniles into the adult population. To gain a thorough understanding of how individuals within populations coordinate their phenological events over ontogeny, it is important to determine the generality of these results for other systems and explore other mechanisms that might be important in driving these inter-stage changes in phenological synchrony.

\section{Implications of phenological synchrony for population dynamics}

Variation in phenological synchrony could have important consequences for population dynamics through its effects on the attributes of offspring cohorts. In this study, hatching synchrony affected survival as well as time to and mass at metamorphosis, which are all attributes known to alter post-metamorphosis performance in amphibians (and other organisms with complex life cycles). At low densities, high hatching synchrony led to high survival, low mass, and similar development time relative to low hatching synchrony, while at high densities, high hatching synchrony lead to similar survival, low mass, and slow development relative to low synchrony (Fig. 1). The combination of metamorph traits that maximizes adult recruitment ultimately depends upon the relative importance of those traits. Some studies of amphibians suggest that development time and/or size at metamorphosis are relatively unimportant for subsequent performance (Beck and Congdon 1999, Boone 2005). If only the number of individuals surviving to metamorphosis matters for B. nebulifer, then at low densities, high hatching synchrony will result in the highest adult recruitment, and at high densities, hatching synchrony might not matter. Other work has demonstrated that size at metamorphosis can have lasting effects (i.e., more than six months later; Goater 1994). If, in addition to survival, size at metamorphosis is important for adult recruitment, then it is difficult to know at low densities whether high or low synchrony exhibit better outcomes, but at high densities, low synchrony would exhibit higher adult recruitment because survival is the same and mass is high compared to high synchrony (Fig. 1). If large metamorphs maintain their size advantage, they can then achieve sexual maturity faster and have higher reproductive fitness. Less is known about the consequences of variation in development time for subsequent survival and growth of amphibians, because they are usually only evaluated shortly after metamorphosis (Beck and Congdon 1999). Many organisms exhibit complex life cycles (e.g., amphibians, insects, fishes, many marine invertebrates), and their life-history events are usually seasonal. If variation in phenological synchrony has similar effects on demographic rates in these other taxa, then this variation could be a key factor driving population dynamics in many communities.

\section{Conclusions}

Recent studies indicate that single metrics (e.g., first or mean timing) frequently provide an insufficient and even misleading view of how phenological distributions shift among years (Miller-Rushing et al. 2010, CaraDonna et al. 2014). For instance, first, mean, and last occurrence of a phenological event frequently shift independently, so variation in one metric cannot readily predict that of another (CaraDonna et al. 2014). This has important implications for estimating the effects of climate change on species. Our results highlight the importance of accounting for other attributes of phenological distributions for understanding the ecological consequences of phenological shifts. We show that variation in synchrony can alter intraspecific competition in ways that affect demographic rates, even when the mean of the distribution remains unchanged. These results emphasize that we need to take a more holistic approach that considers phenological events as temporal distributions with a variety of ecologically important attributes to predict how interannual variation and climate change affect natural communities. 


\section{ACKNOWLEDGMents}

Thanks to Dennis D. Cox, Amy E. Dunham, and Tom E. X. Miller for providing comments on previous versions of the manuscript. We thank Amber Roman, Emily Wheeler, Emily Crowder, and Samantha Masaki for help with data collection. Thanks to the Rice University Department of Ecology and Evolutionary Biology for fellowship funding to N. L. Rasmussen. The work was supported by NSF DEB-0841686 to V. H. W. Rudolf. Study organisms were collected under Scientific Research Permit No. SPR-0409-042 issued by Texas Parks and Wildlife, and all experimental work was conducted in accordance with IACUC protocol no. A10111301.

\section{Literature Cited}

Augspurger, C. K. 1981. Reproductive synchrony of a tropical shrub: experimental studies on effects of pollinators and seed predators on Hybanthus prunifolius (Violaceae). Ecology 62: 775-788.

Ballinger, R. E., and C. O. McKinney. 1966. Developmental temperature tolerance of certain anuran species. Journal of Experimental Zoology 161:21-28.

Bates, D., M. Maechler, B. Bolker, and S. Walker. 2013. lme4: linear mixed-effects models using Eigen and S4. R package version 1.0-4. http://CRAN.R-project.org/package=lme4

Beck, C. W., and J. D. Congdon. 1999. Effects of individual variation in age and size at metamorphosis on growth and survivorship of southern toad (Bufo terrestris) metamorphs. Canadian Journal of Zoology 77:944-951.

Berven, K. A. 1990. Factors affecting population fluctuations in larval and adult stages of the wood frog (Rana sylvatica). Ecology 71:1599-1608.

Blair, W. F. 1960. A breeding population of the Mexican toad (Bufo valliceps) in relation to its environment. Ecology 41: 165-174.

Boone, M. D. 2005. Juvenile frogs compensate for small metamorph size with terrestrial growth: overcoming the effects of larval density and insecticide exposure. Journal of Herpetology 39:416-423.

CaraDonna, P. J., A. M. Iler, and D. W. Inouye. 2014. Shifts in flowering phenology reshape a subalpine plant community. Proceedings of the National Academy of Sciences USA 111: 4916-4921.

DeVito, J. 2003. Metamorphic synchrony and aggregation as antipredator responses in American toads. Oikos 103:75-80.

Domínguez, C. A., and R. Dirzo. 1995. Rainfall and flowering synchrony in a tropical shrub: variable selection on the flowering time of Erythroxylum havanense. Evolutionary Ecology 9:204-216.

Eitam, A., L. Blaustein, and M. Mangel. 2005. Density and intercohort priority effects on larval Salamandra salamandra in temporary pools. Oecologia 146:36-42.

Fox, J. and S. Weisberg. 2011. An R companion to applied regression. Second edition. Sage, Thousand Oaks, California, USA

Geange, S. W., and A. C. Stier. 2009. Order of arrival affects competition in two reef fishes. Ecology 90:2868-2878.

Goater, C. P. 1994. Growth and survival of postmetamorphic toads: interactions among larval history, density, and parasitism. Ecology 75:2264-2274.

Hopper, K. R., P. H. Crowley, and D. Kielman. 1996. Density dependence, hatching synchrony, and within-cohort cannibalism in young dragonfly larvae. Ecology 77:191-200.

Miller-Rushing, A. J., T. T. Hoye, D. W. Inouye, and E. Post. 2010. The effects of phenological mismatches on demography. Philosophical Transactions of the Royal Society B 365: 3177-3186.

Ogutu, J. O., H. P. Piepho, H. T. Dublin, N. Bhola, and R. S. Reid. 2010. Rainfall extremes explain interannual shifts in timing and synchrony of calving in topi and warthog. Population Ecology 52:89-102.

Parmesan, C. 2007. Influences of species, latitudes and methodologies on estimates of phenological response to global warming. Global Change Biology 13:1860-1872.

R Development Core Team. 2013. R: a language and environment for statistical computing. R Foundation for Statistical Computing, Vienna, Austria. www.r-project.org

Rudolf, V. H. W., and M. Singh. 2013. Disentangling climate change effects on species interactions: effects of temperature, phenological shifts, and body size. Oecologia 173:1043-1052.

Semlitsch, R. D., D. E. Scott, and J. H. K. Pechmann. 1988. Time and size at metamorphosis related to adult fitness in Ambystoma talpoideum. Ecology 69:184-192.

Sunahara, T., and M. Mogi. 2002. Priority effects of bamboostump mosquito larvae: influences of water exchange and leaf litter input. Ecological Entomology 27:346-354.

Wilbur, H. M. 1997. Experimental ecology of food webs: complex systems in temporary ponds. Ecology 78:2279-2302.

Wilbur, H. M., and R. A. Alford. 1985. Priority effects in experimental pond communities: responses of Hyla to Bufo and Rana. Ecology 66:1106-1114.

Zuur, A. F., J. M. Hilbe, and E. N. Ieno. 2013. Beginner's guide to GLM and GLMM with R: a frequentist and Bayesian perspective for ecologists. Highland Statistics, Newburgh, UK. 